\title{
Docetaxel-titanate nanotubes enhance radiosensitivity in an androgen-independent prostate cancer model
}

This article was published in the following Dove Press journal:

International Journal of Nanomedicine

30 August 2017

Number of times this article has been viewed

\author{
Céline Mirjolet' \\ Julien Boudon ${ }^{2}$ \\ Alexis Loiseau ${ }^{2}$ \\ Sandy Chevrier' \\ Romain Boidot ${ }^{1}$ \\ Alexandra Oudot ${ }^{3}$ \\ Bertrand Collin ${ }^{3}$ \\ Etienne Martin' \\ Pattayil Alias Joy ${ }^{4}$ \\ Nadine Millot ${ }^{2}$ \\ Gilles Créhange' \\ 'Department of Radiation Oncology, \\ Center Georges-François Leclerc, \\ Dijon, France; ${ }^{2}$ Laboratoire \\ Interdisciplinaire Carnot de \\ Bourgogne, Dijon, France; ${ }^{3}$ Preclinica \\ Imaging Platform, Nuclear Medicine \\ Department, Center Georges- \\ François Leclerc, Dijon, France; \\ ${ }^{4}$ CSIR-National Chemical Laboratory \\ (CSIR-NCL), Pune, India
}

Correspondence: Céline Mirjolet

Department of Radiation Oncology,

Center Georges François Leclerc, I,

rue du Pr Marion, 21079 Dijon, France

$\mathrm{Tel}+33380737518$

Fax +33 380737706

Email cmirjolet@cgl.fr
Abstract: Around 40\% of high-risk prostate cancer patients who undergo radiotherapy (RT) will experience biochemical failure. Chemotherapy, such as docetaxel (DTX), can enhance the efficacy of RT. Multidrug resistance mechanisms often limit drug efficacy by decreasing intracellular concentrations of drugs in tumor cells. It is, therefore, of interest to develop nanocarriers of DTX to maintain the drug inside cancer cells and thus improve treatment efficacy. The purpose of this study was to investigate the use of titanate nanotubes (TiONts) to develop a TiONts-DTX nanocarrier and to evaluate its radiosensitizing in vivo efficacy in a prostate cancer model. In vitro cytotoxic activity of TiONts-DTX was evaluated using an MTS assay. The biodistribution of TiONts-DTX was analyzed in vivo by single-photon emission computed tomography. The benefit of TiONts-DTX associated with RT was evaluated in vivo. Eight groups with seven mice in each were used to evaluate the efficacy of the nanohybrid combined with RT: control with buffer IT injection \pm RT, free DXL \pm RT, TiONts \pm RT and TiONts-DXL \pm RT. Mouse behavior, health status and tumor volume were monitored twice a week until the tumor volume reached a maximum of $2,000 \mathrm{~mm}^{3}$. More than $70 \%$ of nanohybrids were localized inside the tumor $96 \mathrm{~h}$ after administration. Tumor growth was significantly slowed by TiONts-DTX associated with RT, compared with free DTX in the same conditions $(P=0.013)$. These results suggest that TiONts-DTX improved RT efficacy and might enhance local control in high-risk localized prostate cancer.

Keywords: prostate cancer, docetaxel nanocarrier, titanate nanotubes, radiosensitivity, nanoparticle

\section{Introduction}

After initial radiotherapy (RT) for non-metastatic prostate cancer (PCa), between 20\% and $30 \%$ of patients exhibit biochemical relapse. In high-risk localized $\mathrm{PCa}$, hormones combined with RT significantly improve long-term cancer-specific and overall survival rates, but $30 \%$ of these patients still die with a median follow-up of 7.6 years. ${ }^{1}$

Chemotherapy using docetaxel (DTX) is the standard therapy for patients with hormone-refractory PCa. Although the combination of DTX with RT and hormonotherapy (HT) is feasible in high-risk localized PCa, the treatment is highly toxic, which may curb any survival benefit. ${ }^{2,3}$ DTX drives cells into $\mathrm{G}_{2} / \mathrm{M}$ cell cycle arrest by microtubule inhibition. Cells in the G2 or M phase are highly radiosensitive. ${ }^{4}$ However, the clinical applications of DTX are limited both by multidrug resistance mechanisms ${ }^{5}$ and its severe toxic side effects. ${ }^{6}$ Thus, it is of interest to develop carriers of DTX able to maintain the drug inside cancer cells to improve its efficacy and to limit the associated side effects due to systemic circulation. submit your manuscript Dovepress A

in 2 
Nanotechnologies devoted to health have shown considerable growth in terms of innovation and the development of new therapeutic approaches. Nanomaterials and nanohybrids are being developed as contrast agents for magnetic resonance imaging (eg, Combidex ${ }^{\circledR}$ [ferumoxtran-10]), as photosensitizers, ${ }^{7}$ as nanoparticle-based radiosensitizers (gold, gadolinium oxide and so on) ${ }^{8}$ and as nanocarriers to deliver chemotherapies such as liposome-based Myocet ${ }^{\circledR}$ and Caelyx ${ }^{\circledR}$.

Over the past decade, many models of nanocarriers have been developed for diagnostic or therapeutic purposes. Most of them are spherical, and each type has its own chemical and biologic functions and, above all, its own biodistribution kinetics depending on the parameters such as morphology and composition.

More recently, our group evaluated the biologic effects of titanate nanotubes (TiONts) in vitro using glioblastoma cell lines. ${ }^{9}$ These TiONts present new options thanks to their external chemical surface, for example, which allows various molecules to be grafted via the available hydroxyl groups.$^{10}$ Moreover, in contrast to the majority of metal oxide nanoparticles currently developed, TiONts are needle shaped. ${ }^{9}$ TiONts are able to penetrate cells through the possible combination of endocytosis and diffusion with no cytotoxicity and are maintained inside the cells for at least 10 days in vitro. ${ }^{9}$

The purpose of this study was to develop a nanocarrier combining TiONts with DTX (TiONts-DTX) and to evaluate its in vivo biodistribution after intratumoral injection as well as its radiosensitizing efficacy in association with RT in a PCa model.

\section{Materials and methods Development of TiONts-DTX}

First, TiONts were prepared from $\mathrm{TiO}_{2}$ rutile precursor (Ti oxide, at least $99 \%$ pure) by classical hydrothermal synthesis, as described in earlier publications. ${ }^{11,12}$ In the second step, TiONts were modified by 3 -aminopropyl triethoxysilane (APTES) in large excess via hydrolysis and condensation in a solution of water and ethanol (50:50 v:v) under magnetic stirring at $20^{\circ} \mathrm{C}$ for $24 \mathrm{~h}$ to produce TiONts-APTES (hereafter referred to as TiONts- $\left.\mathrm{NH}_{2}\right) \cdot{ }^{10,13,14}$ Then, in the presence of 1-ethyl-3-(dimethylaminopropyl) carbodiimide hydrochloride (10 mol equiv.) and $N$-hydroxysuccinimide (NHS, $20 \mathrm{~mol}$ equiv.), TiONts- $\mathrm{NH}_{2}$ were reacted with PEG-3000 in $0.1 \mathrm{M}$ borate buffer (magnetic stirring, $\mathrm{pH}=8.5$ at $20^{\circ} \mathrm{C}$ for $24 \mathrm{~h}$ ) to generate PEGylated TiONts (TiONts-PEG), as described in Figure 1. Third, DTX activated with $p$-maleimidophenyl isocyanate (PMPI; DTX-PMPI) was mixed with TiONts-PEG to finally yield TiONt-based DTX nanocarriers (TiONtsDTX) using an excess of tris(2-carboxyethyl)-phosphine hydrochloride to reduce disulfides between TiONts-PEG nanohybrids. The reaction took place in PBS $(0.1 \mathrm{M}$ and $\mathrm{pH}=7.4$ ) for $24 \mathrm{~h}$.

All TiONts (bare nanotubes and nanohybrids presented in Figure 1) were purified by dialysis and ultrafiltration (on a $100 \mathrm{kDa}$ MWCO RC [molecular weight cut-off, regenerated cellulose] membrane) with pure water before drying (freeze drying) to eliminate contaminant metals, chemicals and biogenic components. In addition, they were also characterized by transmission electron microscopy (TEM), thermogravimetric analysis (TGA), specific surface area measurements, zeta potential, Fourier transform infrared spectroscopy, X-ray photoelectron spectrometry and Raman spectroscopy.

\section{TiONts-DOTA synthesis and radiolabeling by "'In}

DOTA (1,4,7,10-tetraazacyclododecane-1,4,7,10-tetraacetic acid)-NHS macrocycles were grafted on free amino groups of TiONts-DTX. DOTA-NHS molecules were added to a PBS solution $(0.1 \mathrm{M}$ and $\mathrm{pH}=7.4)$ containing TiONts-DTX under magnetic stirring for $6 \mathrm{~h}$ at $20^{\circ} \mathrm{C}$ to produce single photon emission computed tomography (SPECT-CT)-capable TiONts-DTX (TiONts-DTX-DOTA), as shown in Figure 1. For the in vivo and ex vivo biodistribution studies, DOTA macrocycles were labeled using ${ }^{111} \mathrm{InCl}_{3}$ to yield radiolabeled TiONts-DTX-DOTA $\left[{ }^{11} \mathrm{In}\right]$ nanohybrids. The reaction took place in ammonium acetate $(0.1 \mathrm{M} ; \mathrm{pH}=5.4)$. The solutions were stirred using a thermomixer at $100^{\circ} \mathrm{C}$ for $1 \mathrm{~h}$. The quality of radiolabeling was determined by instant thin layer chromatography, and the yield was $90 \%$ with radiochemical purity higher than $99 \%$ after purification. The absence of free ${ }^{111}$ In was verified with a DTPA (diethylenetriaminepentaacetic acid) challenge test in order to evaluate the labeling stability of TiONts-DTX-DOTA[ $\left.{ }^{111} \mathrm{In}\right]$. Indeed, the results showed excellent stability without transmetalation or the release of radioelements after 5 consecutive days, which is sufficient time to conduct medical imaging.

\section{Cells and animals}

Human PC3 prostate adenocarcinoma cells (ATCC) were cultured in Dulbecco's Modified Eagle's Medium with $10 \%$ fetal bovine serum at $37^{\circ} \mathrm{C}$ and $5 \% \mathrm{CO}_{2}$. Balb/c nude male mice were purchased at 6 weeks of age (Charles River, Saint Germain-Nuelles, France).

Five weeks before the experiments, tumors were induced subcutaneously by injecting $10^{6}$ of PC-3 cells in $200 \mu \mathrm{L}$ 

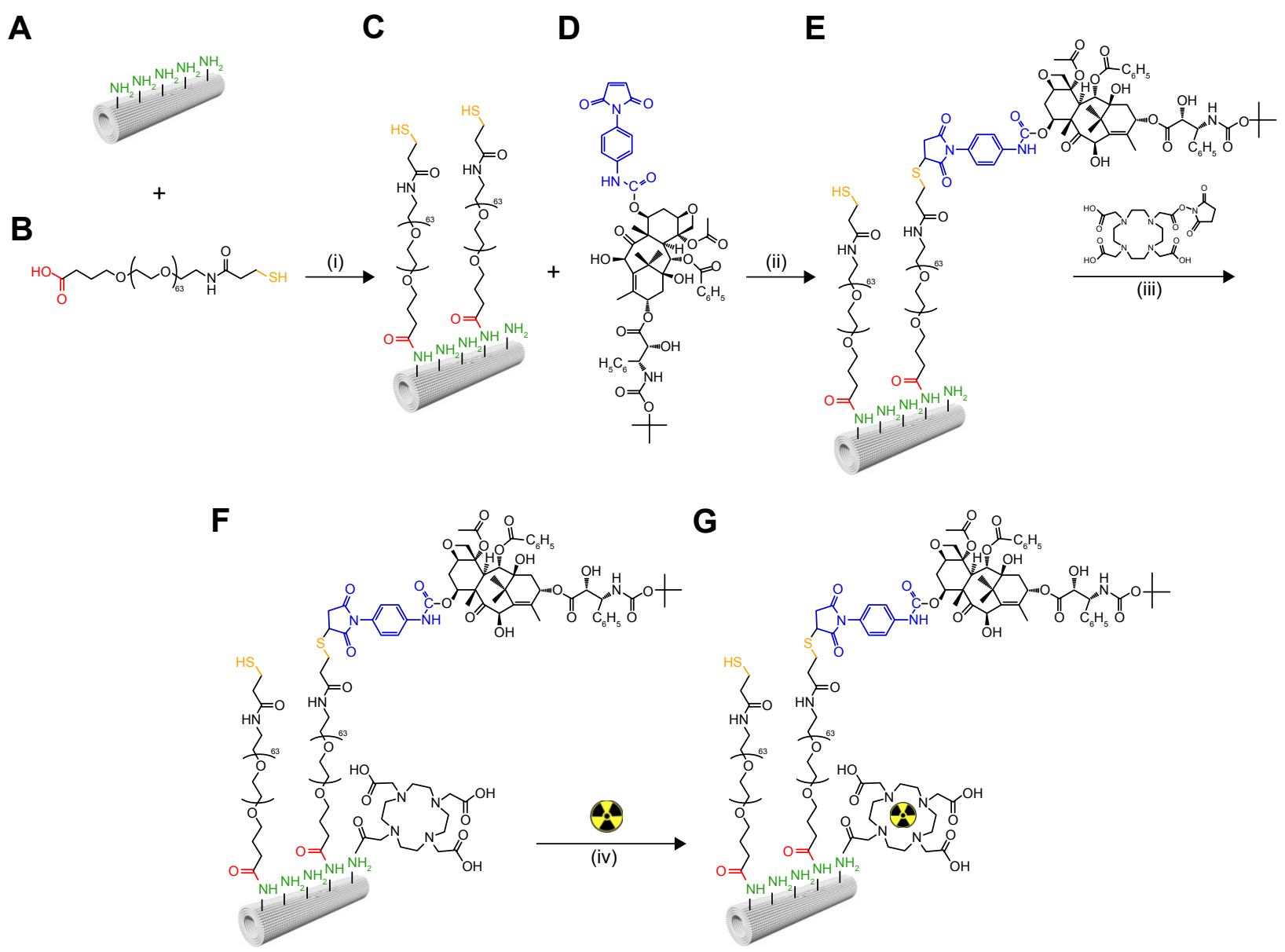

Figure I TiONts-DTX and TiONts-DTX-DOTA["'In] synthesis steps.

Notes: APTES-modified titanate nanotubes (TiONts-APTES) (A) are combined with $\alpha$-acid- $\omega$-thiol-poly (ethylene glycol) (HOOC-PEG-SH, MW =3,000 Da) (B) to generate PEGylated nanotubes (TiONts-PEG-SH) (C) by amide formation. In the second step, (C) is combined with PMPI-modified docetaxel (DTX-PMPI) (D) to finally yield the TiONts-PEG-DTX nanohybrid (E). The nanohybrid (E) is subjected to the reaction of amide formation in the presence of (iii) DOTA-NHS macrocycles to yield nanohybrid (F) (TiONts-DTX-DOTA). In a subsequent step, (F) allows the chelation of indium radionuclides (from "'InCl ${ }_{3}$ ) into DOTA macrocycles to produce SPECT-capable nanohybrid (G) (TiONts-DTX-DOTA[ ${ }^{11}$ In] $)$.

Abbreviations: APTES, 3-aminopropyl triethoxysilane; DTX, docetaxel; SPECT, single-photon emission computed tomography; TiONts, titanate nanotubes; PMPI, p-maleimidophenyl isocyanate.

serum-free culture medium containing Matrigel (50:50, v:v; BD Biosciences) into the right flank of mice. PC-3 tumor cells were implanted $48 \mathrm{~h}$ after whole-body irradiation with a $\gamma$-source (2 Gy, ${ }^{60} \mathrm{Co}$; BioMep, Bretennière, France) was performed in order to improve both tumor development and growth homogeneity. The mice bearing PC-3 tumors were produced by Oncodesign (Dijon, France).

All mice were maintained in specific pathogen-free conditions, and all experiments followed the guidelines of the Federation of European Animal Science Associations. All animal experiments were approved by the Ethics Committee of the University of Burgundy (France).

\section{In vitro evaluation of nanohybrid cytotoxicity}

To evaluate DTX cytotoxicity, the PC-3 cells were seeded in 96-well plates at a concentration of 3,000 cells/well and incubated at $37^{\circ} \mathrm{C}$ in $190 \mu \mathrm{L}$ of drug-free culture medium (DMEM) with $10 \%$ fetal bovine serum for $24 \mathrm{~h}$ before treatment (when the cells were at around $20 \%$ confluence). The cytotoxicity assays were performed with five samples at each concentration of naked TiONts, free DTX, DTX-PMPI or TiONts-DTX. Tumor cells were incubated $(+10 \mu \mathrm{L}$ of drug in $190 \mu \mathrm{L}$ of culture medium) with a range of equivalent DTX concentrations from 0.5 to $500 \mathrm{nM}$ (100 nM of DTX corresponds to $0.35 \mu \mathrm{g}$ of TiONts from TGA). After $96 \mathrm{~h}$ of incubation, cell viability was evaluated using MTS assay (Promega Corporation, Fitchburg, WI, USA) according to Mirjolet et al. ${ }^{15}$

\section{Transmission electron microscopy}

Twenty-four hours after intratumoral administration of TiONts-DTX, three tumors were collected and fixed in 4\% paraformaldehyde and 1.5\% glutaraldehyde. Samples were prepared as previously described to be examined under an H7500 electron microscope (Hitachi Corporation). ${ }^{9}$ 


\section{TiONts-DTX biodistribution analysis}

${ }^{111}$ In was chelated by TiONts-DOTA (see the section on radiolabeling above and Figure 1) to monitor nanohybrid location after in vivo injection into mice using NanoSPECT/CT ${ }^{\circledR}$ small animal imaging tomographic gamma camera (Bioscan Inc.). Fifty microliters of TiONts-DTX-DOTA $\left[{ }^{111} \mathrm{In}\right]$ containing $5 \mathrm{MBq}$ activity (or $50 \mu \mathrm{L}$ of DOTA $\left[{ }^{111} \mathrm{In}\right]$ containing $20 \mathrm{MBq}$ activity) was injected at several points into the tumors of six subcutaneous PC-3 human prostate tumorbearing mice. In vivo biodistribution from 1 to $96 \mathrm{~h}$ after injection was analyzed using SPECT/CT imaging. Seven days postinjection, the animals were killed. The tumor, lungs, liver, kidneys and bladder of each mouse were collected, and the radioactivity in these samples was measured using a gamma counter (Wizard 1480; PerkinElmer).

\section{Evaluation of the efficacy of TiONts-DTX combined with RT}

To evaluate the benefit of nanohybrids combined with RT, $50 \mu \mathrm{L}$ of TiONts-DTX (containing $12 \mu \mathrm{M}$ of DTX grafted onto $10 \mu \mathrm{g}$ of TiONts, corresponding to $60 \mu \mathrm{mol} \mathrm{DTX} / \mathrm{g}$ TiONts in the designed nanohybrid and from TGA measurement) was injected into the tumors. After $24 \mathrm{~h}$ of incubation, the tumors were irradiated with three daily fractions of $4 \mathrm{~Gy}$ using a brachytherapy iridium projector (Microselectron HDR; Elekta).

When the tumors reached a mean tumor volume of around $200 \mathrm{~mm}^{3}$, the mice were randomized according to their individual tumor volume into eight groups (vehicle IT injection \pm RT, DTX \pm RT, TiONts \pm RT and TiONtsDTX \pm RT), with each containing seven mice, using Vivo Manager $^{\circledR}$ software (Biosystemes, Couternon, France).

The behavior and health status of the mice were monitored twice a week, and the tumor volumes were measured using calipers until tumor growth recovery (to a maximum of 2,000 $\mathrm{mm}^{3}$ ). The time for the tumor to reach $1,000 \mathrm{~mm}^{3}$ was assessed for each group and compared using the nonparametric Mann-Whitney $U$ test.

\section{Results}

TiONts were prepared as described in an earlier publication. ${ }^{16}$ They were successfully functionalized to create DTX-bearing nanohybrids using an original step-by-step approach. ${ }^{16}$ The success of each grafting step was verified by different characterization techniques (TEM, TGA, X-ray photoelectron spectrometry, Fourier transform infrared spectroscopy, Raman spectroscopy, zeta potential measurements), and functionalized TiONts were shown to significantly enhance their suspension stability for the targeted biomedical application. ${ }^{16}$ The graft ratios after the addition of APTES, PEGylation, DTX incorporation and DOTA-NHS grafting were determined by TGA. ${ }^{16}$ TiONts-DTX-DOTA presented 2.1 DOTA $/ \mathrm{nm}^{2}$ and $0.2 \mathrm{DTX} / \mathrm{nm}^{2}$.

\section{In vitro cytotoxicity assessment}

Free DTX presented the greatest cytotoxic effect with an $\mathrm{IC}_{50}$ parameter evaluated at $\sim 2-3 \mathrm{nM}$. The PMPI-activated DTX induced a decrease in cytotoxic activity, leading to an $\mathrm{IC}_{50}$ of 15-20 nM for this compound. As expected, the TiONts-DTX finally presented the lowest cytotoxic activity $\left(\mathrm{IC}_{50}\right.$ evaluated at 350-400 $\mathrm{nM}$; Figure 2).

In these technical conditions, naked TiONts did not exhibit toxicity in PC-3 cells.

Similar results were observed with another prostate cancer cell line (22RV1, data not shown).

\section{Biodistribution analysis}

The biodistribution kinetics assessed using SPECT-CT imaging showed that $1 \mathrm{~h}$ after intratumoral administration, a significantly higher amount of TiONts-DTX-DOTA $\left[{ }^{111} \mathrm{In}\right]$ activity (94.0\% [mean $\{88.4 \%-99.7 \%\}]$ of the administrated dose) was retained inside the tumor as compared to DOTA- ${ }^{111}$ In vehicle (only 3.8\% [mean $\{0.6 \%-5.8 \%\}]$ tumor retention). A mean of $72.9 \%([70.3 \%-75.4 \%])$ of TiONts-DTX-DOTA $\left[{ }^{111} \mathrm{In}\right]$ was maintained inside the tumor $96 \mathrm{~h}$ after intratumoral injection (Figure 3A2), whereas DOTA $\left[{ }^{111} \mathrm{In}\right]$ vehicle was totally absent from the tumor $3 \mathrm{~h}$ postinjection (Figure $3 \mathrm{~A} 1$ ). The quantity of TiONts-DTX localized inside the tumor decreased slowly between 3 and 96 h after injection (Figure 3A2). From $24 \mathrm{~h}$ after

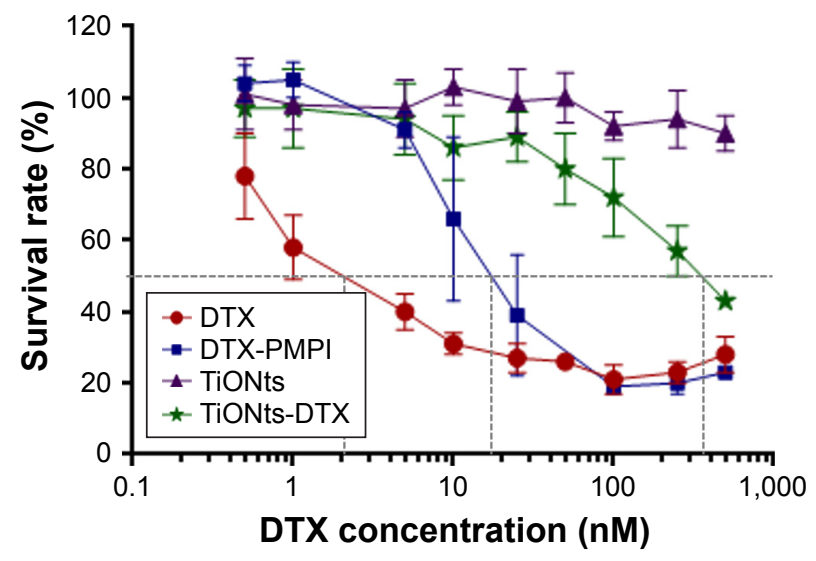

Figure 2 TiONts-DTX cytotoxic activity evaluation.

Notes: PC-3 cells were treated with free DTX, activated DTX (DTX-PMPI), DTX grafted to TiONts (TiONts-DTX) or bare TiONts (concentration equivalent to TiONtsDTX) for $96 \mathrm{~h}$. Each point corresponds to the mean values of five replicates \pm SD (results shown are representative curves from two independent experiments).

Abbreviations: DTX, docetaxel; TiONts, titanate nanotubes; PMPI, p-maleimidophenyl isocyanate. 


\section{A1}
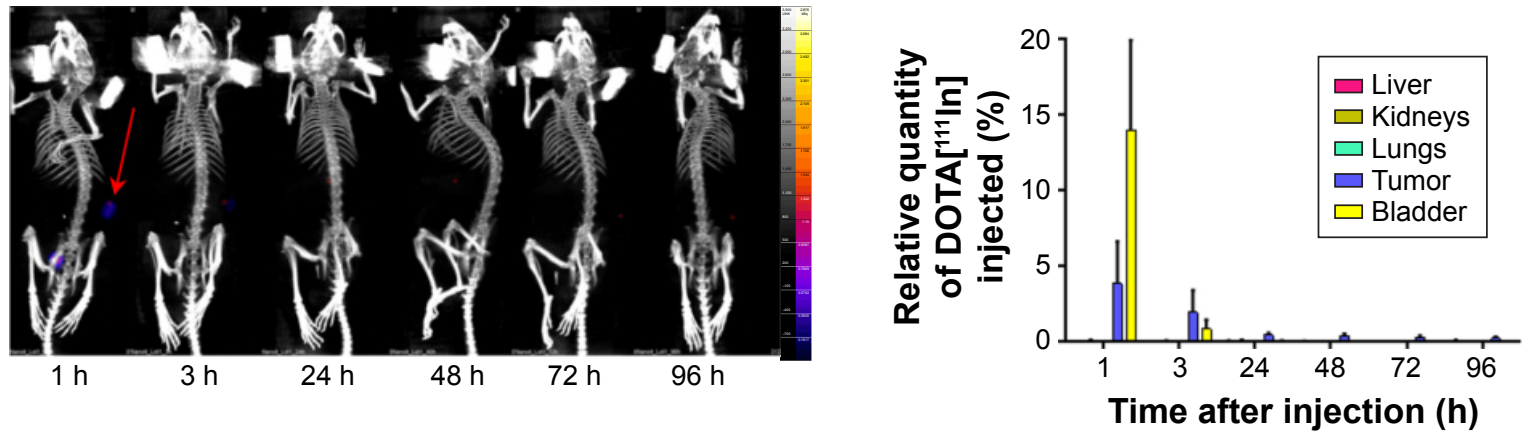

A2
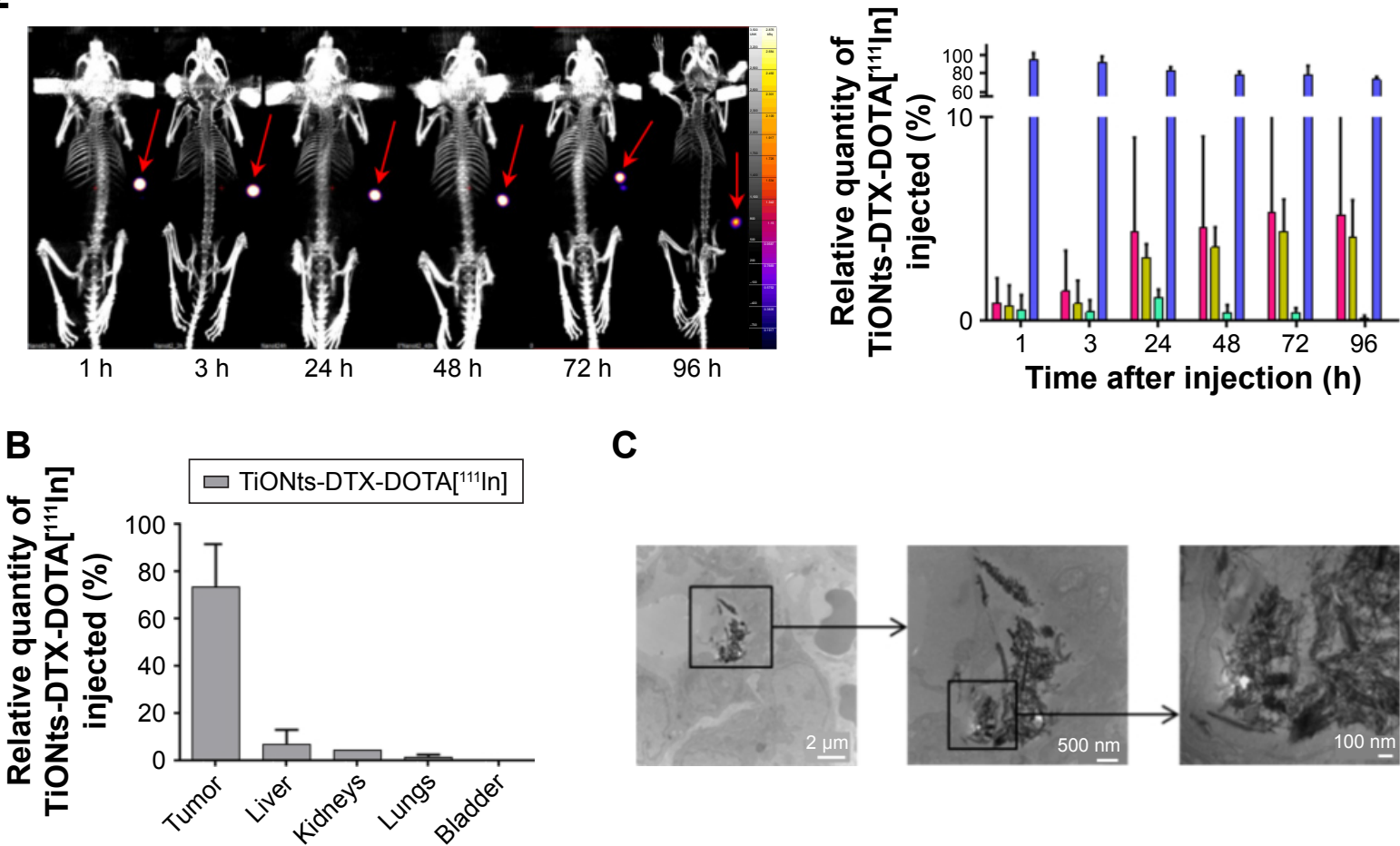

C

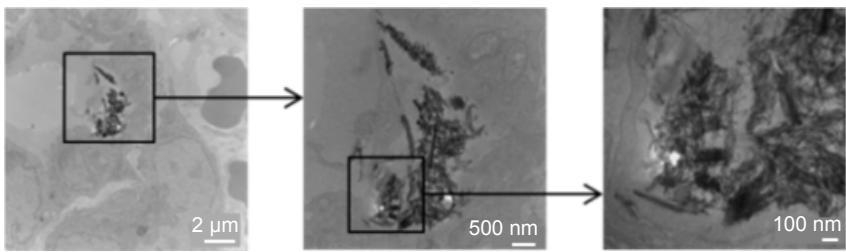

Figure 3 TiONts-DTX biodistribution analysis.

Notes: (A) SPECT-CT imaging of kinetics and biodistribution analysis for each organ (expressed as a percentage of injected "'In activity, taking into account the decrease in "'In activity after the injection of DOTA["'In] (AI) or TiONts-DTX-DOTA["'In] (A2)). (B) TiONts-DTX-DOTA["'In] biodistribution in dissected organs by radioactivity detection using gamma counting 7 days after injection (mean value \pm SD). (C) TEM images showing the intracellular location of TiONts-DTX $24 \mathrm{~h}$ after injection into PC-3 tumors.

Abbreviations: DTX, docetaxel; SPECT-CT, single-photon emission computed tomography-computed tomography; TEM, transmission electron microscopy; TiONts, titanate nanotubes.

injection, TiONts-DTX were detected in moderate quantities inside the liver (from 4.4\% [mean $\{1.1 \%-7.6 \%\}$ ] of TiONtsDTX injected at $24 \mathrm{~h}$ to $5.2 \%$ [mean $\{1.3 \%-9.1 \%\}]$ at $96 \mathrm{~h}$ ) and kidney (from 3.1\% [mean $\{2.6-3.8\}$ ] of TiONts-DTX injected at $24 \mathrm{~h}$ to $4.1 \%$ [mean $\{2.5 \%-6.1 \%\}$ ] at $96 \mathrm{~h}$ ). The quantity of TiONts-DTX detected within the lungs was $<1.2 \%$ of the dose of TiONts-DTX administered.

Seven days after the injection, $73.0 \%$ (mean $[60.0 \%-$ $86.0 \%])$ of TiONts-DTX-DOTA $\left[{ }^{111} \mathrm{In}\right]$ was still localized inside the tumor (Figure 3B), 6.2\% in the liver (mean [1.9$10.5]$ ), $3.9 \%$ in the kidney (mean [3.9-3.9]) and $0.8 \%$ in the lung (mean [0-1.5]).

\section{Efficacy of TiONts-DTX combined with RT}

TEM analysis showed that during RT administration $(24 \mathrm{~h}$ after injection), TiONts-DTX were localized inside the cells (Figure 3C) and more particularly in the cytoplasm.

Tumor volumes of control, placebo, free DTX and naked TiONts groups were comparable. Three of the four groups that received RT presented slowed tumor growth compared with the first four groups without RT (Figure 4).

Interestingly, mice treated with TiONts-DTX without RT exhibited a trend toward slowed tumor growth to reach a volume of $1,000 \mathrm{~mm}^{3}$, compared with mice receiving free 


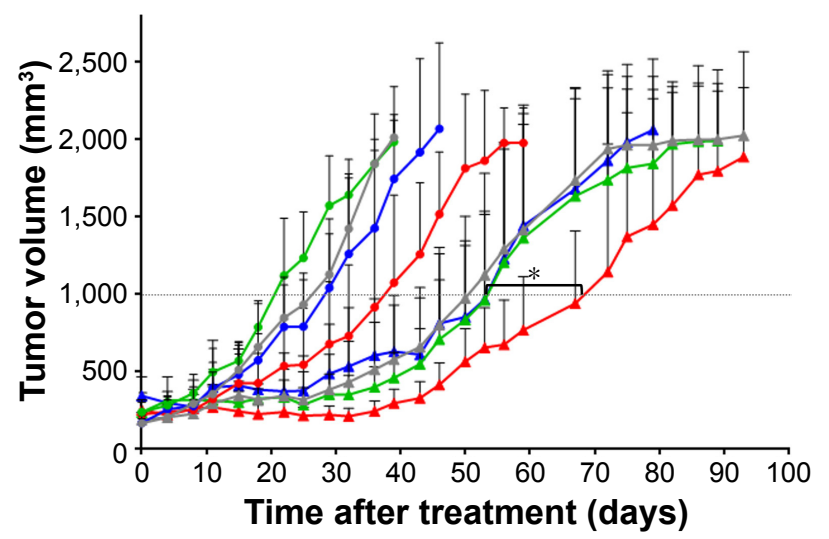

$\rightarrow$ Vehicle $\rightarrow$ TiONts $\rightarrow$ DTX $\rightarrow$ TiONts-DTX
$\neq$ Vehicle + RT + TiONts + RT $\rightarrow$ DTX + RT
$\rightarrow$ TiONts-DTX + RT

Figure 4 Therapeutic effect of vehicle, free DTX, free TiONts, and TiONts-DTX associated or not with RT administered with three daily fractions of $4 \mathrm{~Gy}, 24 \mathrm{~h}$ after injection into PC-3 xenografted tumors.

Notes: Data are mean values of tumor volumes $\pm S D$ ( $n=7$ per treatment group, eight treatment groups). ${ }^{*} P=0.013$ (TiONts-DTX + RT vs DTX + RT or TiONts + RT), comparison performed using nonparametric Mann-Whitney test.

Abbreviations: DTX, docetaxel; RT, radiotherapy; TiONts, titanate nanotubes.

DTX (40.5 days [median $\{15.0-50.0\}$ ] vs 30.8 days [median $\{21.3-49.5\}]$, respectively; $P=0.11$ ).

Free DTX associated with RT did not present any effect since the tumor growth curves for DTX + RT, bare TiONts + RT and placebo + RT groups overlapped.

Mice receiving TiONts-DTX associated with RT exhibited significantly slowed tumor growth to reach a volume of $1,000 \mathrm{~mm}^{3}$, compared with mice receiving free DTX + RT (73.7 days [median $\{58.9-89.0\}$ ] vs 56.0 days [median $\{31.5-74.0\}$ ], respectively; $P=0.013$; Figure 4). According to the effect of TiONts-DTX and RT alone, we can conclude that TiONts-DTX and RT induce an additive effect.

\section{Discussion}

In this study, we highlighted that our nanotube DTX carrier (TiONts-DTX) was able to stay inside the tumor for several days after intratumor injection. Moreover, its association with RT delayed tumor growth even further.

DTX is able to arrest cells in the radiosensitive $G_{2} / M$ phase of the cell cycle and can increase radiosensitivity by a factor of 2.5. ${ }^{17}$ Several trials have evaluated tolerance to DTX combined with hormonal therapy and RT for high-risk PCa. ${ }^{2,18,19}$ The results of a Phase II trial showed that although feasible, $30 \%$ of Grade 3 or higher toxicities was observed. ${ }^{2}$ More recently, the GETUG-12 trial found improved relapsefree survival with sequential DTX combined with HT and RT compared with HT alone. ${ }^{18}$ As a consequence, it seems interesting to develop chemoradiation treatments in high-risk $\mathrm{PCa}$, as they may improve the therapeutic ratio by decreasing systemic toxicities while maintaining a high radiosensitizing effect when delivered concomitantly.

The clinical applications of DTX remain limited because of multidrug resistance mechanisms and its known toxic side effects. ${ }^{6,20}$ Therefore, in order to avoid the abovementioned drawbacks of free DTX, we aimed to develop a safe nanoparticle-based DTX carrier to administer the drug directly into prostate tumors and to keep it there for longer.

In a previous study, we demonstrated that TiONts remained within glioma cancer cells for more than 10 days. ${ }^{9}$ Thus, these interesting characteristics of nanotubes make them ideal chemo-nanocarriers capable of keeping a drug inside tumors.

Recently, Kumar et al also associated DTX to silica nanoparticles, carried by needles of brachytherapy spacers for PCa. ${ }^{21}$ In their device, DTX was not linked to the spacers and was delivered inside the tumor. So far, these authors have only demonstrated the feasibility of using their device by a preliminary in vivo study. Its efficacy, when used in association with RT, has still to be demonstrated. Unlike the strategy chosen by Kumar et al, we developed TiONts-based nanocarriers that remained linked to DTX molecules. DTX stays within the tumor as long as these needle-shaped nanoparticles remain within tumor cells too.

In spite of the diminished cytotoxic activity of TiONtsDXT compared with free DTX, we decided to evaluate this nanohybrid on the assumption that TiONts would keep the DTX inside the tumor for longer and would thus have a longer cytotoxic activity than free DTX. However, in a future study, we will modify PEG length to try to improve this activity, that is, to facilitate DTX-tubulin interactions.

The slowed tumor growth shown in Figure 4 seems very interesting because only $12 \mu \mathrm{M}$ of DTX was injected, which is equivalent to $25 \mu \mathrm{g} / \mathrm{kg}$. These quantities were 200 times less than those used in other studies $(5 \mathrm{mg} / \mathrm{kg}$ for polymeric nanoparticles containing DTX). ${ }^{22}$ In addition, $12 \mu \mathrm{M}$ of DTX would correspond to a dose of $75 \mu \mathrm{g} / \mathrm{m}^{2}$ (for a $20 \mathrm{~g}$ mouse with a body surface area of $66 \mathrm{~cm}^{2}$ ), as compared with $\sim 75 \mathrm{mg} / \mathrm{m}^{2}$ for IV injections of DTX in humans.

Concerning the curves for the TiONTs-DTX + RT and DTX + RT groups, we found that tumor growth was significantly delayed in the group treated with the nanocarrier. However, we found no obvious difference between these two groups during the first 40 days after treatment. Ionizing radiation can induce instantaneous death, but also delays death (clonogenic death). This death is induced after the 
activation of inefficient DNA repair mechanisms. In clinical situations, the effects of RT may be seen several weeks after the end of the treatment. This is why tumor growth in the TiONts-DTX + RT groups slowed down.

By the in vivo approach, we demonstrated that the TiONts-DTX group presented a trend toward better treatment efficacy compared with the free DTX group. This trend could be explained by DTX retention inside tumor cells by TiONts. For similar reasons, TiONts-DTX, compared with free DTX, significantly improved the RT efficacy.

In a previous study, TiONts were injected IV. In this first biodistribution study, we found a higher accumulation of TiONts inside the lungs and liver $1 \mathrm{~h}$ after IV injection, but this decreased rapidly. These accumulations thus decreased via the elimination of TiONts in the urine ( 4 and $24 \mathrm{~h}$ in the lung and liver, respectively). ${ }^{23}$ These results highlighted that the localization of TiONts in the lung and liver was not definitive, and the tubes could be eliminated. However, before starting a clinical trial, an in-depth analysis of the biodistribution kinetics of TiONts-DTX over a longer period seems necessary to evaluate the total clearance of nanohybrids and to overcome the accumulation of TiONts inside healthy organs.

To improve TiONts-DTX + RT efficacy before clinical development, we are considering increasing the quantity of DTX grafted onto nanoparticles and adding gold nanoparticles. This new study aims to improve the efficacy of RT even further using gold, which has a high atomic number $(Z=79)$ and can thus amplify the photoelectric effect. ${ }^{24}$

Obviously, the next step for the development of our gold-DTX-nanocarrier will be a new biodistribution study using later time kinetics to ensure that TiONts do not remain within healthy organs.

After these preclinical results, we may hope to improve progression-free survival in our patients with locally advanced prostate cancer.

To conclude, this new nanocarrier remained within the tumor for several days and improved RT efficacy. So, it could be injected in men harboring high-risk localized $\mathrm{PCa}$ using prostate brachytherapy needles, as previously described by Quivrin et $\mathrm{al},{ }^{25}$ in order to enhance radiation response. Ultrasound/magnetic resonance imaging fusion during the brachytherapy procedure may also guide the physician in injecting our nanocarrier focally in the index lesion. ${ }^{25}$

\section{Acknowledgments}

This work was supported by the "Ligue Contre le Cancer GE” (Comités 21, 25). The authors would like to thank
CheMatech for providing DOTA macrocycles. They also thank Philip Bastable, who is a native English speaker and proof reader for the Research Unit at Dijon University Hospital, for his review and corrections of the wording of this manuscript.

\section{Disclosure}

The authors report no conflicts of interest in this work.

\section{References}

1. D'Amico AV, Chen MH, Renshaw AA, Loffredo M, Kantoff PW. Androgen suppression and radiation vs radiation alone for prostate cancer: a randomized trial. JAMA. 2008;299(3):289-295.

2. Bolla M, Hannoun-Levi JM, Ferrero JM, et al. Concurrent and adjuvant docetaxel with three-dimensional conformal radiation therapy plus androgen deprivation for high-risk prostate cancer: preliminary results of a multicentre phase II trial. Radiother Oncol. 2010;97(2):312-317.

3. MarshallDT, Ramey S, Golshayan AR, Keane TE, Kraft AS, Chaudhary U. Phase I trial of weekly docetaxel, total androgen blockade, and imageguided intensity-modulated radiotherapy for localized high-risk prostate adenocarcinoma. Clin Genitourin Cancer. 2014;12(2):80-86.

4. Redpath JL, Sun C. Sensitivity of a human hybrid cell line (HeLa X skin fibroblast) to radiation-induced neoplastic transformation in G2, M, and mid-G1 phases of the cell cycle. Radiat Res. 1990;121(2):206-211.

5. Armstrong CM, Gao AC. Drug resistance in castration resistant prostate cancer: resistance mechanisms and emerging treatment strategies. Am J Clin Exp Urol. 2015;3(2):64-76.

6. Bissett D, Setanoians A, Cassidy J, et al. Phase I and pharmacokinetic study of taxotere (RP 56,976) administered as a 24-hour infusion. Cancer Res. 1993;53(3):523-527.

7. Couleaud P, Morosini V, Frochot C, Richeter S, Raehm L, Durand JO. Silica-based nanoparticles for photodynamic therapy applications. Nanoscale. 2010;2(7):1083-1095.

8. Retif P, Pinel S, Toussaint M, et al. Nanoparticles for radiation therapy enhancement: the key parameters. Theranostics. 2015;5(9): 1030-1044.

9. Mirjolet C, Papa AL, Crehange G, et al. The radiosensitization effect of titanate nanotubes as a new tool in radiation therapy for glioblastoma: a proof-of-concept. Radiother Oncol. 2013;108(1):136-142.

10. Papa AL, Boudon J, Bellat V, et al. Dispersion of titanate nanotubes for nanomedicine: comparison of PEI and PEG nanohybrids. Dalton Trans. 2015;44(2):739-746.

11. Kasuga T, Hiramatsu M, Hoson A, Sekino T, Niihara K. Titania nanotubes prepared by chemical processing. Adv Mat. 1999;11(15):1307-1311.

12. Papa AL, Maurizi L, Vandroux D, Walker P, Millot N. Synthesis of titanate nanotubes directly coated with USPIO in hydrothermal conditions: a new detectable nanocarrier. J Phys Chem C. 2011;115(39): 19012-19017.

13. Paris J, Bernhard Y, Boudon J, Heintz O, Millot N, Decreau RA. Phthalocyanine-titanate nanotubes: a promising nanocarrier detectable by optical imaging in the so-called imaging window. RSC Adv. 2015; 5(9):6315-6322.

14. Pontón PI, d'Almeida JRM, Marinkovic BA, et al. The effects of the chemical composition of titanate nanotubes and solvent type on 3-aminopropyltriethoxysilane grafting efficiency. App Surf Sci. 2014; 301:315-322.

15. Mirjolet JF, Barberi-Heyob M, Merlin JL, et al. Thymidylate synthase expression and activity: relation to S-phase parameters and 5-fluorouracil sensitivity. Br J Cancer. 1998;78(1):62-68.

16. Loiseau A, Boudon J, Mirjolet C, Créhange G, Millot N. Taxane-grafted metal-oxide nanoparticles as a new theranostic tool against cancer: the promising example of docetaxel-functionalized titanate nanotubes in preclinical development on prostate tumors. Adv Healthc Mater. Epub 2017 May 18. 
17. Cooper BT, Sanfilippo NJ. Concurrent chemoradiation for high-risk prostate cancer. World J Clin Oncol. 2015;6(4):35-42.

18. Fizazi K, Faivre L, Lesaunier F, et al. Androgen deprivation therapy plus docetaxel and estramustine vs androgen deprivation therapy alone for high-risk localized prostate cancer (GETUG 12): a phase 3 randomized controlled trial. Lancet Oncol. 2015;16(7):787-794.

19. Rosenthal SA, Hunt D, Sartor AO, et al. A phase 3 trial of 2 years of androgen suppression and radiation therapy with or without adjuvant chemotherapy for high-risk prostate cancer: final results of radiation therapy oncology group phase 3 randomized trial NRG oncology RTOG 9902. Int J Radiat Oncol Biol Phys. 2015;93(2):294-302.

20. Zhu Y, Liu C, Armstrong C, Lou W, Sandher A, Gao AC. Antiandrogens inhibit ABCB1 efflux and ATPase activity and reverse docetaxel resistance in advanced prostate cancer. Clin Cancer Res. 2015; 21(18):4133-4142.

21. Kumar R, Belz J, Markovic S, et al. Nanoparticle-based brachytherapy spacers for delivery of localized combined chemoradiation therapy. Int J Radiat Oncol Biol Phys. 2015;91(2):393-400.
22. Hrkach J, Von Hoff D, Mukkaram Ali M, et al. Preclinical development and clinical translation of a PSMA-targeted docetaxel nanoparticle with a differentiated pharmacological profile. Sci Transl Med. 2012; 4(128):128ra39.

23. Boudon J, Papa AL, Paris J, Millot N. Titanate nanotubes as a versatile platform for nanomedicine. In: Seifalian A, De Mel A, Kalaskar DM, editors. Nanomedicine. 2014:403-427.

24. Schuemann J, Berbeco R, Chithrani DB, et al. Roadmap to Clinical use of gold nanoparticles for radiation sensitization. Int J Radiat Oncol Biol Phys. 2016;94(1):189-205.

25. Quivrin M, Loffroy R, Cormier L, et al. Multiparametric MRI and post implant CT-based dosimetry after prostate brachytherapy with iodine seeds: The higher the dose to the dominant index lesion, the lower the PSA bounce. Radiother Oncol. 2015;117(2):258-261.
International Journal of Nanomedicine

\section{Publish your work in this journal}

The International Journal of Nanomedicine is an international, peerreviewed journal focusing on the application of nanotechnology in diagnostics, therapeutics, and drug delivery systems throughout the biomedical field. This journal is indexed on PubMed Central, MedLine, CAS, SciSearch ${ }^{\circledR}$, Current Contents ${ }^{\circledR} /$ Clinical Medicine,

\section{Dovepress}

Journal Citation Reports/Science Edition, EMBase, Scopus and the Elsevier Bibliographic databases. The manuscript management system is completely online and includes a very quick and fair peer-review system, which is all easy to use. Visit http://www.dovepress.com/ testimonials.php to read real quotes from published authors. 